\title{
Bronchial casts associated with hilar lymphatic and pulmonary lymphoid abnormalities
}

\author{
J WIGGINS, E SHEFFIELD, P K JEFFERY, D M GEDDES, B CORRIN
}

From the Brompton Hospital, London

\begin{abstract}
A patient who has expectorated bronchial casts for 23 years is reported. The casts contained mucus, fibrin, and lymphocytes, and the resected middle lobe showed dilated lymphatics with thickened walls at the hilum and lymphoid aggregates in the terminal bronchioles.
\end{abstract}

We describe an unusual patient who has expectorated bronchial casts for 23 years but who shows no evidence of aspergillosis, asthma, or other disease. Lymphatic and lymphoid abnormalities found in his lung may underlie the disorder.

\section{Case report}

A previously fit house builder first presented in 1964 at the age of 26 with episodic chest tightness accompanied and often relieved by the expectoration of stringy sputum. He had smoked 10 cigarettes daily until two years previously. The sputum, which was easily coughed up, was white and occasionally contained fresh blood. There was no dyspnoea. The episodes lasted several days, the intervening "dry" periods varying from a few days to a few weeks, when the patient was completely normal. Examination of the expectorate showed that it consisted of casts up to $6 \mathrm{~cm}$ long, with a branching pattern corresponding to the bronchial tree (fig 1). One expectorate of casts would fill a Petri dish. Clinical examination, chest radiography, rigid bronchoscopy, bronchography and a pulmonary angiogram showed nothing abnormal. No treatment was given.

Over the succeeding years he continued to complain of episodic expectoration, which he found socially embarrassing. During this time no abnormality was found on clinical examination. The full blood count, haemoglobin concentration, erythrocyte sedimentation rate, serum immunoglobulin concentrations, chest radiographs, lung volumes, gas transfer, and serial peak expiratory flow all remained within normal limits. There was no serological evidence of aspergillus infection and the aspergillus skinprick test response was negative. Only bacterial commensals were cultured from 12 specimens of sputum. No fungal or bacterial pathogens were isolated.

In 1978 fibreoptic bronchoscopy showed mild inflamma-

Address for reprint requests: Professor B Corrin, Brompton Hospital, London SW3 6HP.

Accepted 22 December 1988 tion of the right middle lobe orifice, from which a cast was $\overrightarrow{\vec{x}}$ easily aspirated. Bronchial mucosa obtained at biopsy was unremarkable. Further bronchograms were normal, but a $₫$ ventilation-perfusion scan indicated reduced ventilation of $\omega$ the right middle lobe. Cyclical antibiotics and nebulised $N$ saline and bronchodilators afforded no relief, and in 1979 the

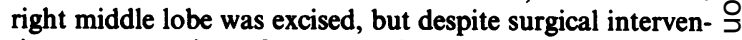
tion expectoration of casts continued. A further cast was removed from the left lower lobe bronchus in 1983, after which the airways appeared normal. Again, no histological lesion was seen in biopsy material. A trial of prednisolone $\supset$ $40 \mathrm{mg}$ daily for one month and a prolonged course of $\vec{\omega}$ nebulised carbocysteine provided no relief.

The patient is now $\mathbf{4 9}$ years old and his symptoms have continued unchanged for 23 years. The results of all laboratory investigations remain normal and a third fibreoptic bronchoscopy, bronchoalveolar lavage, and investigation of ciliary function have all failed to reveal any abnormality. Because of the lymphocytic nature of the casts, he was $\mathbb{D}$ prescribed $100 \mathrm{mg}$ of cyclophosphamide a day for three months, but this had no effect on his expectoration. He has developed no other symptom or illness, has normal lung function, and remains in full time employment.

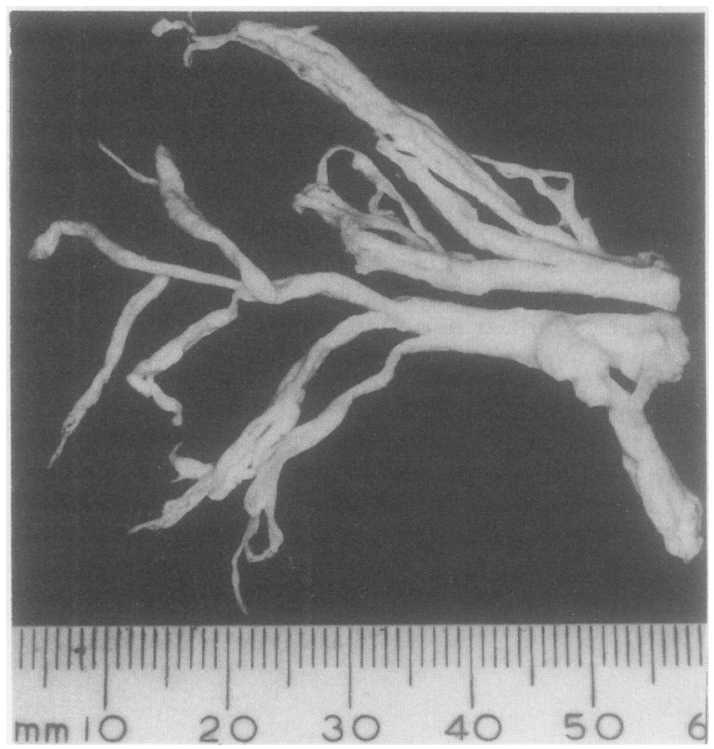

Fig 1 One of the bronchial casts. 


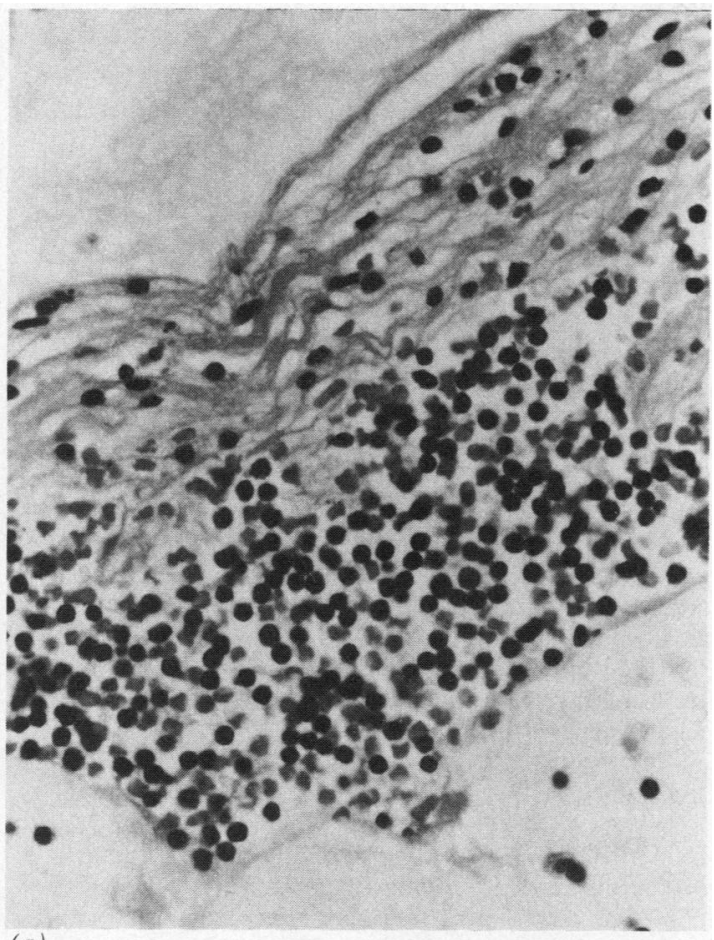

(a)

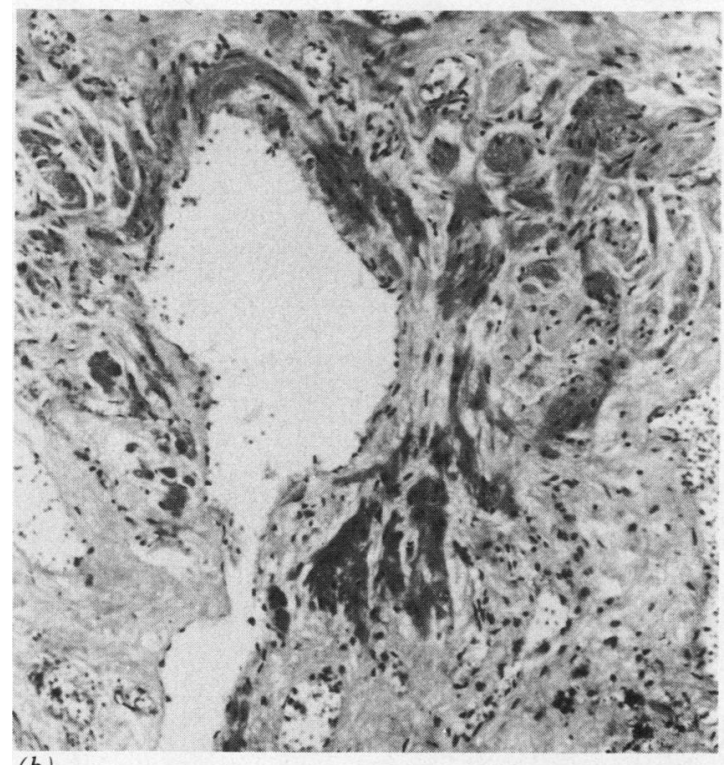

(b)

Fig 2 (a) One of the bands of fibrin, containing many lymphocytes flanked by bands of mucus. (b) Hilar fibrosis with dilated lymphatics showing muscular hypertrophy.

\section{PATHOLOGICAL FINDINGS}

Many casts were examined microscopically and one was studied in particular detail. Light microscopy showed it to consist of alternating layers of acidic mucus and fibrin, the latter containing numerous mature lymphocytes but no eosinophils (fig $2(a)$ ). Mixed bacterial colonies were evident in the outer layers of mucus; no fungi were identified. Immunocytochemical examination showed that about $70 \%$ of the lymphocytes were $\mathrm{T}$ cells and that there were small numbers of B cells. Scanning electron microscopy confirmed the lamellar nature of the cast, and the presence of lymphocytes in the fibrin and rod shaped and filamentous bacteria in the mucoid component. Transmission electron microscopy showed wavy, electron dense fibrils resembling fibrin mixed with lymphocytes.

The airway anatomy of the resected middle lobe was normal on gross examination, but there was fibrosis at the hilum. Histologically, the fibrotic area contained many dilated lymphatic vessels; the walls of the larger lymphatics were thickened by muscular hyperplasia (fig $2(b)$ ). The fibrosis and abnormal lymphatics were confined to the hilum; the rest of the lobe was normal apart from lymphoid aggregates, which comprised roughly equal numbers of $\mathbf{B}$ and $\mathrm{T}$ lymphocytes, in the region of the terminal bronchioles. Bronchus associated lymphoid tissue was otherwise unremarkable, as were the bronchial glands and surface epithelium.

\section{Discussion}

This condition does not appear to have been described previously. The main features are chronic episodic expectoration of bronchial casts containing large numbers of $T$ lymphocytes, hyperplastic lymphoreticular aggregates around terminal bronchioles, and a lymphatic abnormality at the hilum of the lung. The cellular content of the casts in the present case distinguishes them from the mucus plugs of asthma and plastic bronchitis, which are characterised by exfoliated epithelial cells and eosinophils rather than lymphocytes.' The large numbers of lymphocytes in the casts and the lymphoid hyperplasia within the lung suggest that the disorder described here is a response to an as yet unidentified persistent antigenic stimulus. The bacteria identified in the casts are assumed to be saprophytic.

The fibrolymphatic abnormality at the hilum may have contributed to the serous component of the casts by promoting the transudation of lymph into the airways. It is uncertain whether it represents a developmental anomaly of the hilar lymphatics or is an acquired scar that has obstructed these vessels. The hypertrophied muscle in their walls is evidence of such obstruction. If responsible for the casts, the hilar abnormality must be bilateral, as the casts have been observed bronchoscopically in both lungs and have continued to form after excision of the middle lobe.

\section{Reference}

1 Sanerkin NG, Seal RME, Leopold JG. Plastic bronchitis, mucoid impaction of the bronchi and allergic boncho-pulmonary aspergillosis, and their relationship to bronchial asthma. Ann Allergy 1966:24:586-94. 